\title{
Benefits and Risks of Antiretroviral Therapy for Perinatal HIV Prevention
}

\author{
M.G. Fowler, M. Qin, S.A. Fiscus, J.S. Currier, P.M. Flynn, T. Chipato, J. Mclntyre, \\ D. Gnanashanmugam, G.K. Siberry, A.S. Coletti, T.E. Taha, K.L. Klingman, \\ F.E. Martinson, M. Owor, A. Violari, D. Moodley, G.B. Theron, R. Bhosale, \\ R. Bobat, B.H. Chi, R. Strehlau, P. Mlay, A.J. Loftis, R. Browning, T. Fenton, \\ L. Purdue, M. Basar, D.E. Shapiro, and L.M. Mofenson, \\ for the IMPAACT 1077BF/1077FF PROMISE Study Team*
}

A BSTRACT

The authors' full names, academic degrees, and affiliations are listed in the Appendix. Address reprint requests to Dr. Fowler at the Department of Pathology, Johns Hopkins University School of Medicine, 600 N. Wolfe St., 443 Carnegie, Baltimore, MD 21287, or at mgfowler@mujhu.org.

*A complete list of members of the International Maternal Pediatric Adolescent AIDS Clinical Trials Network (IMPAACT) 1077BF/1077FF Promoting Maternal and Infant Survival Everywhere (PROMISE) Study Team is provided in the Supplementary Appendix, available at NEJM.org.

N Engl J Med 2016;375:1726-37. DOI: 10.1056/NEJMoa1511691 Copyright (c) 2016 Massachusetts Medical Society.

\section{BACKGROUND}

Randomized-trial data on the risks and benefits of antiretroviral therapy (ART) as compared with zidovudine and single-dose nevirapine to prevent transmission of the human immunodeficiency virus (HIV) in HIV-infected pregnant women with high CD4 counts are lacking.

METHODS

We randomly assigned HIV-infected women at 14 or more weeks of gestation with CD4 counts of at least 350 cells per cubic millimeter to zidovudine and single-dose nevirapine plus a 1-to-2-week postpartum "tail" of tenofovir and emtricitabine (zidovudine alone); zidovudine, lamivudine, and lopinavir-ritonavir (zidovudine-based ART); or tenofovir, emtricitabine, and lopinavir-ritonavir (tenofovir-based ART). The primary outcomes were HIV transmission at 1 week of age in the infant and maternal and infant safety.

\section{RESULTS}

The median CD4 count was 530 cells per cubic millimeter among 3490 primarily black African HIV-infected women enrolled at a median of 26 weeks of gestation (interquartile range, 21 to 30). The rate of transmission was significantly lower with ART than with zidovudine alone $(0.5 \%$ in the combined ART groups vs. $1.8 \%$; difference, -1.3 percentage points; repeated confidence interval, -2.1 to -0.4$)$. However, the rate of maternal grade 2 to 4 adverse events was significantly higher with zidovudine-based ART than with zidovudine alone $(21.1 \%$ vs. $17.3 \%, \mathrm{P}=0.008)$, and the rate of grade 2 to 4 abnormal blood chemical values was higher with tenofovir-based ART than with zidovudine alone ( $2.9 \%$ vs. $0.8 \%, \mathrm{P}=0.03$ ). Adverse events did not differ significantly between the ART groups ( $\mathrm{P}>0.99)$. A birth weight of less than $2500 \mathrm{~g}$ was more frequent with zidovudine-based ART than with zidovudine alone $(23.0 \%$ vs. $12.0 \%, \mathrm{P}<0.001)$ and was more frequent with tenofovir-based ART than with zidovudine alone ( $16.9 \%$ vs. $8.9 \%, \mathrm{P}=0.004)$; preterm delivery before 37 weeks was more frequent with zidovudine-based ART than with zidovudine alone (20.5\% vs. $13.1 \%, \mathrm{P}<0.001)$. Tenofovir-based ART was associated with higher rates than zidovudine-based ART of very preterm delivery before 34 weeks $(6.0 \%$ vs. $2.6 \%, \mathrm{P}=0.04)$ and early infant death ( $4.4 \%$ vs. $0.6 \%, \mathrm{P}=0.001)$, but there were no significant differences between tenofovir-based ART and zidovudine alone ( $\mathrm{P}=0.10$ and $\mathrm{P}=0.43$ ). The rate of HIVfree survival was highest among infants whose mothers received zidovudine-based ART.

CONCLUSIONS

Antenatal ART resulted in significantly lower rates of early HIV transmission than zidovudine alone but a higher risk of adverse maternal and neonatal outcomes. (Funded by the National Institutes of Health; PROMISE ClinicalTrials.gov numbers, NCT01061151 and NCT01253538.) 
NTIRETROVIRAL REGIMENS USED FOR the prevention of mother-to-child transmission of the human immunodeficiency virus (HIV) have evolved from the first successful trial that used zidovudine single-drug prophylaxis in 1994 to current triple-drug regimens., ${ }^{1,2}$ Although there are clear benefits of combination antiretroviral therapy (ART) for the mother and infant, these do not come without risks; some studies have shown higher rates of adverse pregnancy outcomes with maternal ART than with regimens containing fewer antiretroviral agents..$^{3-5}$ The Promoting Maternal and Infant Survival Everywhere (PROMISE) trial compared the relative efficacy and safety of various proven antiretroviral strategies for the prevention of mother-tochild transmission during pregnancy among asymptomatic HIV-infected pregnant women with high CD4 counts. Results evaluating early transmission through the week 1 postpartum trial visit (in utero, intrapartum, or very early breastfeeding transmission) are presented.

\section{METHODS}

\section{TRIAL SITES AND PARTICIPANTS}

The PROMISE trial was conducted at 14 sites in seven countries (India, Malawi, South Africa, Tanzania, Uganda, Zambia, and Zimbabwe). When enrollment began in 2011, standard prevention of mother-to-child transmission for women with a CD4 count of more than 350 cells per cubic millimeter in these countries was zidovudine with intrapartum single-dose nevirapine and a 1-to-2-week "tail" of two nucleosides to prevent maternal nevirapine resistance. ${ }^{6}$

Eligibility criteria included a CD4 count of at least 350 cells per cubic millimeter (or a countryspecific threshold for initiating triple-drug ART, if that threshold was higher), gestation of at least 14 weeks and not in labor, no previous use of triple-drug ART, no clinical or immune-related indication for triple-drug ART, a hemoglobin level of at least $7.5 \mathrm{~g}$ per deciliter, an absolute neutrophil count of at least 750 cells per cubic millimeter, an alanine aminotransferase level of less than 2.5 times the upper limit of the normal range, an estimated creatinine clearance of more than $60 \mathrm{ml}$ per minute, and no serious pregnancy complications. Receipt of one or two antiretroviral agents for the prevention of motherto-child transmission in previous pregnancies and for 30 days or fewer during the current pregnancy before enrollment was permitted. Key exclusion criteria were active tuberculosis or receipt of tuberculosis treatment within 30 days before trial entry, hepatitis B virus (HBV) infection requiring $\mathrm{HBV}$ treatment (patients who did not require HBV treatment could enroll), a structural or conduction heart defect, or a fetus with a serious congenital malformation.

All pregnant women provided written informed consent. The trial was approved by local and collaborating institutional review boards and reviewed every 6 months by an independent data and safety monitoring board (members are listed in the Supplementary Appendix, available with the full text of this article at NEJM.org).

\section{TRIAL DESIGN AND TREATMENT REGIMENS}

The PROMISE trial compared open-label strategies and included sequential randomizations: antepartum randomization (at 14 weeks of gestation or later) to one of three regimens, with postpartum randomization (breast-feeding settings) at 6 to 14 days to maternal ART or infant prophylaxis during breast-feeding and "maternal health" randomization of women receiving ART to continue or stop ART after breast-feeding cessation or birth (formula-feeding settings). Breastfeeding settings (enrollment started in April 2011) and formula-feeding settings (enrollment started in July 2011) had separate protocols (available at NEJM.org); the complete PROMISE trial schema is shown in Figure S1A and S1B in the Supplementary Appendix. At the November 2014 planned interim analysis, the prespecified early stopping guideline for efficacy for the antepartum component was met, and the data and safety monitoring board recommended releasing the results.

In the antepartum component, women were randomly assigned to one of three regimens: zidovudine plus intrapartum single-dose nevirapine with 6 to 14 days of tenofovir and emtricitabine post partum (zidovudine alone); zidovudine, lamivudine, and lopinavir-ritonavir (zidovudinebased ART); or tenofovir, emtricitabine, and lopinavir-ritonavir (tenofovir-based ART). All regimens were continued through 6 to 14 days post partum. All infants received nevirapine from birth until postpartum randomization. Figure 1 shows the antepartum regimens and dosing.

Antiretroviral agents were donated by AbbVie, 


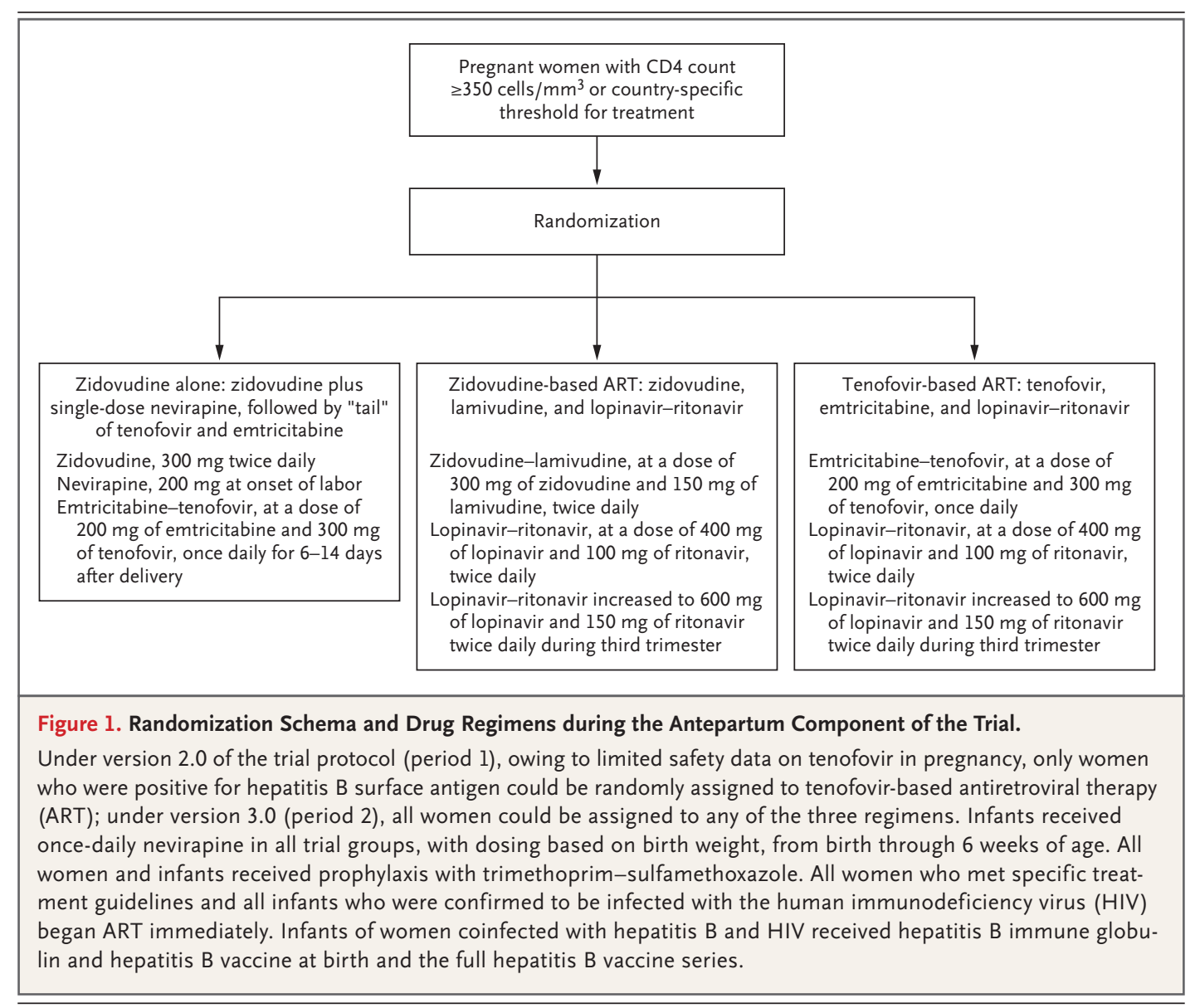

Boehringer Ingelheim, Gilead Sciences, and ViiV Healthcare/GlaxoSmithKline, with the exception that some nevirapine was purchased from Boehringer Ingelheim at a discounted rate by a contractor to the Eunice Kennedy Shriver National Institute of Child Health and Human Development for use in the trial. None of the pharmaceutical companies had any role in the trial design, data collection, data analyses, or manuscript preparation.

When the trial began, there were limited safety data on tenofovir in pregnancy, and randomization to tenofovir-based ART was limited to women coinfected with HIV and HBV, because benefit was felt to outweigh risk in that group; comparative analyses including the group assigned to tenofovir-based ART were to be limited to a nested HBV substudy. During period 1 (April 2011 through September 2012), women without HBV coinfection were assigned only to zidovudine alone or zidovudine-based ART. However, in October 2012, with increased data on tenofovir in pregnancy, the protocol was modified to allow women regardless of HBV status to be assigned to any of the three regimens, with an analytic plan to compare safety across all three groups for participants enrolled during period 2 (October 2012 through October 1, 2014).

\section{TRIAL PROCEDURES}

Screening included confirmation of maternal HIV status and measurement of the CD4 count and HBV surface antigen (HBsAg) level. Maternal visits occurred at trial entry; at weeks 2, 4, 8, and 12 and then every 4 weeks until delivery; at labor and delivery; and at postpartum week 1 (6 to 14 days). A complete blood count and blood chemical values (alanine aminotransferase and creatinine levels and calculated creatinine clearance) were obtained at trial entry; at weeks 4, 8, and 12 and then every 8 weeks; at delivery; and at postpartum week 1 . The plasma HIV RNA concentration (quantified by real-time polymerasechain-reaction assay in the ART groups and by 
batch testing of stored samples in the zidovudine-alone group) was measured at trial entry, week 4 , and delivery or postpartum week 1 . The CD4 count was obtained at week 12, delivery, and postpartum week 1 . Infant visits, including HIV nucleic acid testing and measurement of safety laboratory values, occurred at birth (to 5 days of age) and week 1 (6 to 14 days of age). Full details are provided in the protocol.

\section{EFFICACY AND SAFETY OUTCOMES}

Early infant HIV infection, the primary efficacy outcome, was defined as a positive infant HIV nucleic-acid-testing result on the birth or week 1 specimen, confirmed by a second positive result on a separate specimen obtained on a different day (as soon as possible after the first positive result); tests were performed in laboratories that were certified by the Virology Quality Assurance Program of the Division of AIDS (DAIDS) of the National Institute of Allergy and Infectious Diseases. Infant HIV-free survival, a secondary safety outcome, was defined as an infant being alive and not HIV-infected through the week 1 visit.

The DAIDS Table for Grading the Severity of Adult and Pediatric Adverse Events was used to grade adverse events. ${ }^{7}$ Primary safety analyses were composite, with secondary analysis of individual components. For mothers, the composite outcome was grade 2 or higher hematologic abnormalities or abnormal blood chemical values or grade 3 or higher signs or symptoms during pregnancy through week 1 post partum. For pregnancy, the composite outcome was a low birth weight of less than $2500 \mathrm{~g}$, a preterm delivery before 37 weeks of gestation (according to the Ballard examination, when available, or obstetrical estimate), spontaneous abortion $(<20$ weeks of gestation), stillbirth ( $\geq 20$ weeks of gestation), or a congenital anomaly (according to the criteria of the Metropolitan Atlanta Congenital Defects Program of the Centers for Disease Control and Prevention). Severe adverse pregnancy outcomes included a very low birth weight of less than $1500 \mathrm{~g}$, a very preterm delivery before 34 weeks, spontaneous abortion, stillbirth, or a major congenital anomaly. For infants, the composite outcome was death from any cause, grade 3 or higher hematologic abnormalities or abnormal blood chemical values, or grade 3 or higher signs or symptoms through week 1 post partum.

\section{TRIAL ROLES}

The PROMISE trial was designed by the protocol team, with the first author and the cochairs taking the lead. The clinical sites gathered the data; the International Maternal Pediatric Adolescent AIDS Clinical Trials Network (IMPAACT) Statistical and Data Analysis Center at the Harvard T.H. Chan School of Public Health analyzed the data according to the agreed-on statistical plans and with input from the protocol team and the data and safety monitoring board. All the authors vouch for the completeness and accuracy of the data and analyses and for the fidelity of the trial to the protocol.

\section{STATISTICAL ANALYSIS}

Randomization was stratified according to HBV status and country. The target sample was 4400 mother-infant sets, 3400 from breast-feeding settings and 1000 from formula-feeding settings, to be combined for analysis. We calculated that this sample size would provide the trial with more than $90 \%$ power to detect a cumulative early transmission difference of $4 \%$ in the zidovudine-alone group versus $2 \%$ in the two ART groups combined, with a 5\% (two-sided) type I error, allowing for $10 \%$ loss to follow-up and two interim efficacy analyses, and $95 \%$ power to detect differences as small as $25 \%$ versus $20 \%$ for safety outcomes.

The a priori analysis plan required combining the results of the two ART groups for efficacy analyses because efficacy was expected to be similar. However, because adverse events were expected to differ between ART groups, safety analyses (including HIV-free survival through week 1) were to include pairwise comparisons of all three groups.

Safety comparisons of the zidovudine-alone group and the group assigned to zidovudinebased ART were based on data from both period 1 and period 2. Pairwise safety comparisons of the group assigned to tenofovir-based ART with the zidovudine-alone group or the group assigned to zidovudine-based ART were restricted to data from participants enrolled during period 2, when there was concurrent randomization to all three groups. All analyses were conducted on an intention-to-treat basis. The unit of analysis was the mother-infant set for efficacy analyses and the individual participant (mother or infant) for safety analyses. For multiple-gestation births, 
perinatal transmission was defined as confirmed HIV infection in any of the live-born siblings; for infant safety analyses, each live-born sibling was included separately.

Categorical variables were compared between groups with Fisher's exact test. A two-sided P value of less than 0.05 was considered to indicate statistical significance for all analyses except efficacy. Interim efficacy analyses used a group-sequential repeated confidence interval for the difference in the early transmission rate with the use of the Lan-DeMets approach with an O'Brien-Fleming type I error spending function (for this interim analysis, the confidence coefficient was $96.5 \%$, to preserve an experiment-wise type I error rate of $5 \%$ ). If the repeated confidence interval excluded zero, this would indicate a significant difference. One prespecified subgroup analysis and two post hoc subgroup analyses were performed, with interaction tests assessing heterogeneity of treatment effects.

\section{RESULTS}

BASELINE MATERNAL CHARACTERISTICS

From April 2011 through September 10, 2014 (the cutoff date for the interim analysis), we enrolled 3529 mother-infant sets (3245 in breast-feeding settings and 284 in formula-feeding settings). A total of 39 women were excluded from analyses: 3 were determined not to be pregnant at enrollment, 2 had molar pregnancies, and 34 were coinfected with HIV and HBV and were randomly assigned to receive tenofovir-based ART during period 1.

Of the 3490 mother-infant sets included in analyses, $2261(65 \%)$ were enrolled during period 1 and 1229 (35\%) during period 2. There were 3202 live births (1045 during period 2), including 57 multiple births with at least 1 liveborn infant (24 during period 2). The numbers and flow of participants in the trial are shown in Figure S2A (randomization to zidovudine alone or zidovudine-based ART in periods 1 and 2) and Figure S2B (randomization to zidovudine alone, zidovudine-based ART, or tenofovir-based ART in period 2 only) in the Supplementary Appendix.

Baseline maternal characteristics were well balanced between the zidovudine-alone group and the group assigned to zidovudine-based ART (periods 1 and 2) and between the group assigned to tenofovir-based ART and each of the other two trial groups (period 2) (Table 1; Table S1 in the Supplementary Appendix provides further details on CD4 cell count and viral-load quantification according to group). There were differences between periods 1 and 2 in country-specific enrollment (higher during period 1 in South Africa and during period 2 in Zimbabwe and Uganda) and in the percentage of women receiving zidovudine alone during the current pregnancy before enrollment (19\% vs. $28 \%$ ). Most women were African, young (median age, 26 years), and asymptomatic ( $97 \%$ had World Health Organization [WHO] had clinical stage $1 \mathrm{HIV}$ infection). At screening, the median CD4 count was 530 cells per cubic millimeter, and $3 \%$ of the women were HBsAg-positive.

\section{EFFICACY AND HIV-FREE SURVIVAL RESULTS (THROUGH WEEK I AFTER DELIVERY)}

The rate of early transmission was significantly lower in the combined maternal ART groups than in the zidovudine-alone group $(0.5 \%$ vs. $1.8 \%$; difference, -1.3 percentage points; repeated confidence interval, -2.1 to -0.4 ; no significant heterogeneity in subgroup analyses) (Table 2). The rate of infant HIV-free survival through week 1 was significantly higher (i.e., the rate of early transmission or death was significantly lower) with zidovudine-based ART than with zidovudine alone (periods 1 and 2) (Fig. 2A) or tenofovir-based ART (period 2) (Fig. 2B).

\section{SAFETY RESULTS IN THE ANTEPARTUM COMPONENT Maternal Adverse Events}

During periods 1 and 2, women receiving zidovudine-based ART had a significantly higher rate of any grade 2 or higher adverse event than those receiving zidovudine alone $(21.1 \%$ vs. $17.3 \%$, $\mathrm{P}=0.008$ ) and a higher rate of grade 2 or higher abnormal blood chemical values (primarily elevations in the alanine aminotransferase level) ( $5.8 \%$ vs. $1.3 \%, \mathrm{P}<0.001$ ) (Table 3 , and Table S2 in the Supplementary Appendix). During period 2 , women receiving tenofovir-based ART had a significantly higher rate of grade 2 or higher abnormal blood chemical values than those receiving zidovudine alone ( $2.9 \%$ vs. $0.8 \%, \mathrm{P}=0.03$ ), but there was no significant difference between the two ART groups $(\mathrm{P}=0.26)$. Grade 2 or higher elevations in the creatinine level occurred in less than $1 \%$ of women in all groups. Table S3 in the Supplementary Appendix provides additional de- 


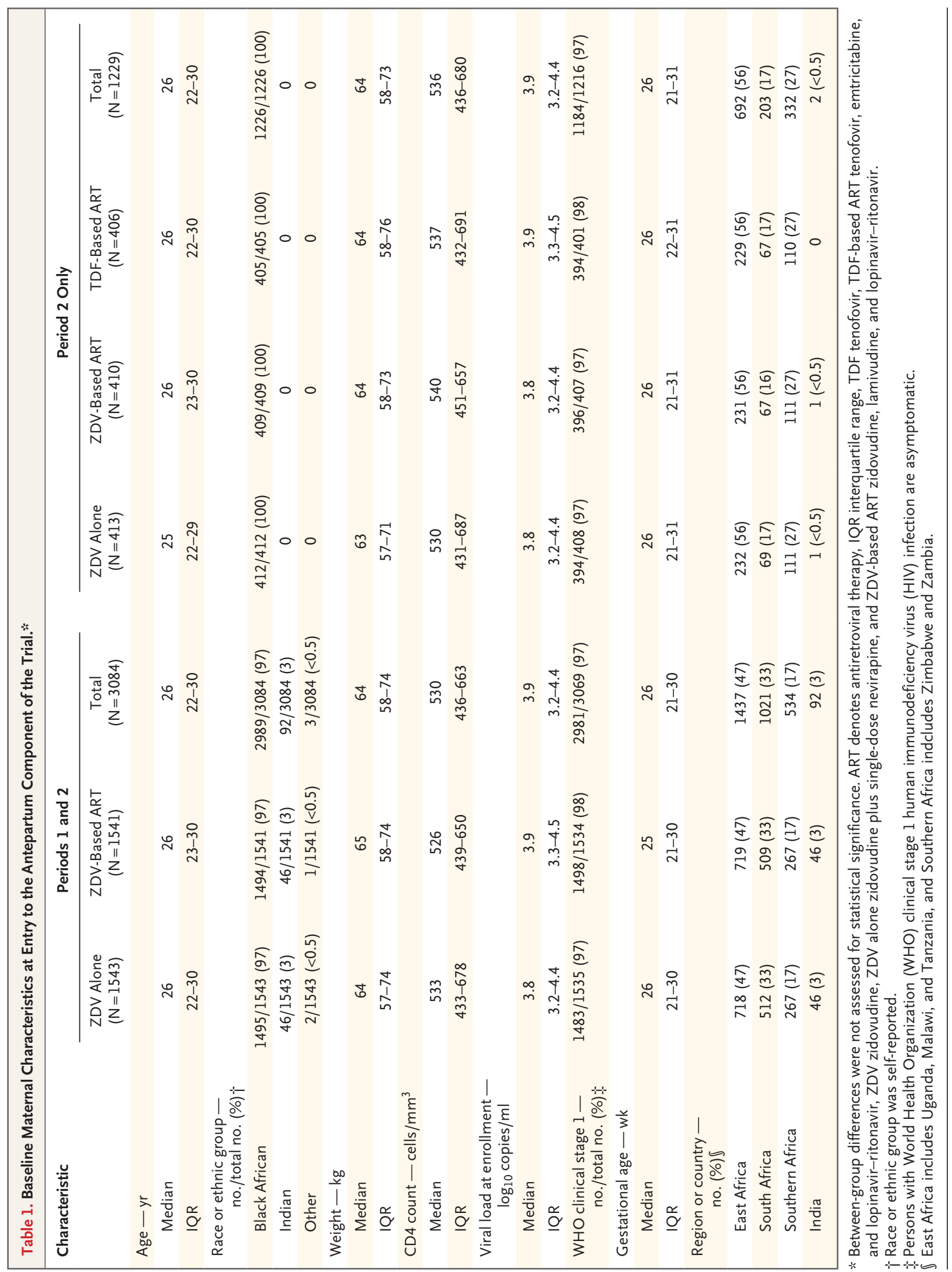

N ENGLJ MED 375;18 NeJM.ORG NOVEMBER 3, 2016 


\begin{tabular}{|c|c|c|c|c|c|}
\hline \multirow[t]{2}{*}{ Subgroup } & $\begin{array}{l}\text { ZDV } \\
\text { Alone }\end{array}$ & $\begin{array}{l}\text { ZDV-Based } \\
\text { ART }\end{array}$ & $\begin{array}{l}\text { TDF-Based } \\
\text { ART }\end{array}$ & $\begin{array}{l}\text { Difference, ZDV-Based ART and } \\
\text { TDF-Based ART vs. ZDV Alone }\end{array}$ & $\begin{array}{l}\text { P Value for } \\
\text { Interaction }\end{array}$ \\
\hline & \multicolumn{3}{|c|}{ no. of mother-infant sets/total no. (\%) } & percentage points (repeated CI) & \\
\hline All mother-infant sets & $25 / 1386(1.8)$ & $7 / 1385(0.5)$ & $2 / 325(0.6)$ & $-1.3(-2.1$ to -0.4$)$ & \\
\hline Maternal gestational age at trial entry $\uparrow$ & & & & & 0.68 \\
\hline$<34 \mathrm{wk}$ & $16 / 1229(1.3)$ & $6 / 1230(0.5)$ & $1 / 274(0.4)$ & $-0.8(-1.6$ to -0.1$)$ & \\
\hline$\geq 34 \mathrm{wk}$ & $9 / 157(5.7)$ & $1 / 154(0.6)$ & $1 / 51(2.0)$ & $-4.8(-8.9$ to -0.6$)$ & \\
\hline Maternal CD4 count at trial entry & & & & & 0.70 \\
\hline $350-499$ cells $/ \mathrm{mm}^{3}$ & $16 / 577(2.8)$ & $4 / 592(0.7)$ & $1 / 136(0.7)$ & $-2.1(-3.7$ to -0.5$)$ & \\
\hline$\geq 500$ cells $/ \mathrm{mm}^{3}$ & $9 / 809$ (1.1) & $3 / 793(0.4)$ & $1 / 189(0.5)$ & -0.7 (-1.6 to 0.2$)$ & \\
\hline Maternal viral load at trial entry & & & & & 0.22 \\
\hline$<1000$ copies/ml & $0 / 299$ & $1 / 253(0.4)$ & $0 / 57$ & $0.3(-0.4$ to 1.0$)$ & \\
\hline$\geq 1000$ copies $/ \mathrm{ml}$ & $25 / 1083(2.3)$ & $6 / 1129(0.5)$ & $2 / 268(0.7)$ & $-1.7(-2.8$ to -0.7$)$ & \\
\hline Missing data & 4 & 3 & 0 & & \\
\hline
\end{tabular}

* The analysis of infant HIV infection according to maternal gestational age at trial entry was a prespecified analysis; the other two subgroup analyses were post hoc analyses. $\mathrm{Cl}$ denotes confidence interval, and HIV human immunodeficiency virus.

$\uparrow$ Data on maternal gestational age at trial entry were missing for one woman in the group assigned to ZDV-based ART.

tails on signs, symptoms, and diagnoses. There were no maternal deaths. The rate of trial-drug discontinuation was low (2 to $5 \%$ ) and did not differ significantly among the three groups; $4 \%$ of the women (120 of 3248) discontinued their assigned antiretroviral regimen prematurely, and $0.7 \%$ had a discontinuation that was assessed as being possibly related to a trial drug.

\section{Adverse Pregnancy Outcomes}

During periods 1 and 2, women receiving zidovudine-based ART had significantly higher rates than women receiving zidovudine alone of adverse pregnancy outcomes $(40.0 \%$ vs. $27.5 \%$, $\mathrm{P}<0.001)$, a low birth weight of less than $2500 \mathrm{~g}$ ( $23.0 \%$ vs. $12.0 \%, \mathrm{P}<0.001)$, and preterm delivery before 37 weeks $(20.5 \%$ vs. $13.1 \%, \mathrm{P}<0.001)$ (Table 3; Table S2 in the Supplementary Appendix provides additional details on the combined outcome of low birth weight and preterm delivery and the combined outcome of very low birth weight and very preterm delivery). During period 2, women receiving tenofovir-based ART had significantly higher rates than women receiving zidovudine alone of adverse outcomes $(34.7 \%$ vs. $27.2 \%, P=0.04$ ) and a low birth weight of less than $2500 \mathrm{~g}(16.9 \%$ vs. $8.9 \%, \mathrm{P}=0.004)$, but there was no significant difference for any outcome between the two ART groups. No significant differences in stillbirth or spontaneous abortion and congenital anomalies were observed among the three groups (Table S4 in the Supplementary Appendix lists congenital anomalies).

With respect to severe pregnancy outcomes, no significant differences were observed for the composite or individual outcomes between women receiving zidovudine-based ART and those receiving zidovudine alone (periods 1 and 2). During period 2, no significant differences between tenofovir-based ART and zidovudine alone were observed for composite or individual outcomes. However, women receiving tenofovir-based ART had significantly higher rates than those receiving zidovudine-based ART of severe adverse pregnancy outcomes $(9.2 \%$ vs. $4.3 \%, \mathrm{P}=0.02)$ and very preterm delivery before 34 weeks (6.0\% vs. $2.6 \%$, $\mathrm{P}=0.04$ ); in the group assigned to zidovudine-

Figure 2 (facing page). Probability of Infant HIV Infection or Death through the Week 1 Postpartum Visit (6 to 14 Days after Delivery).

The insets show the same data on an expanded $y$ axis. TDF denotes tenofovir, TDF-based ART tenofovir, emtricitabine, and lopinavir-ritonavir, ZDV zidovudine, ZDV alone zidovudine plus single-dose nevirapine, and ZDVbased ART zidovudine, lamivudine, and lopinavir-ritonavir. 


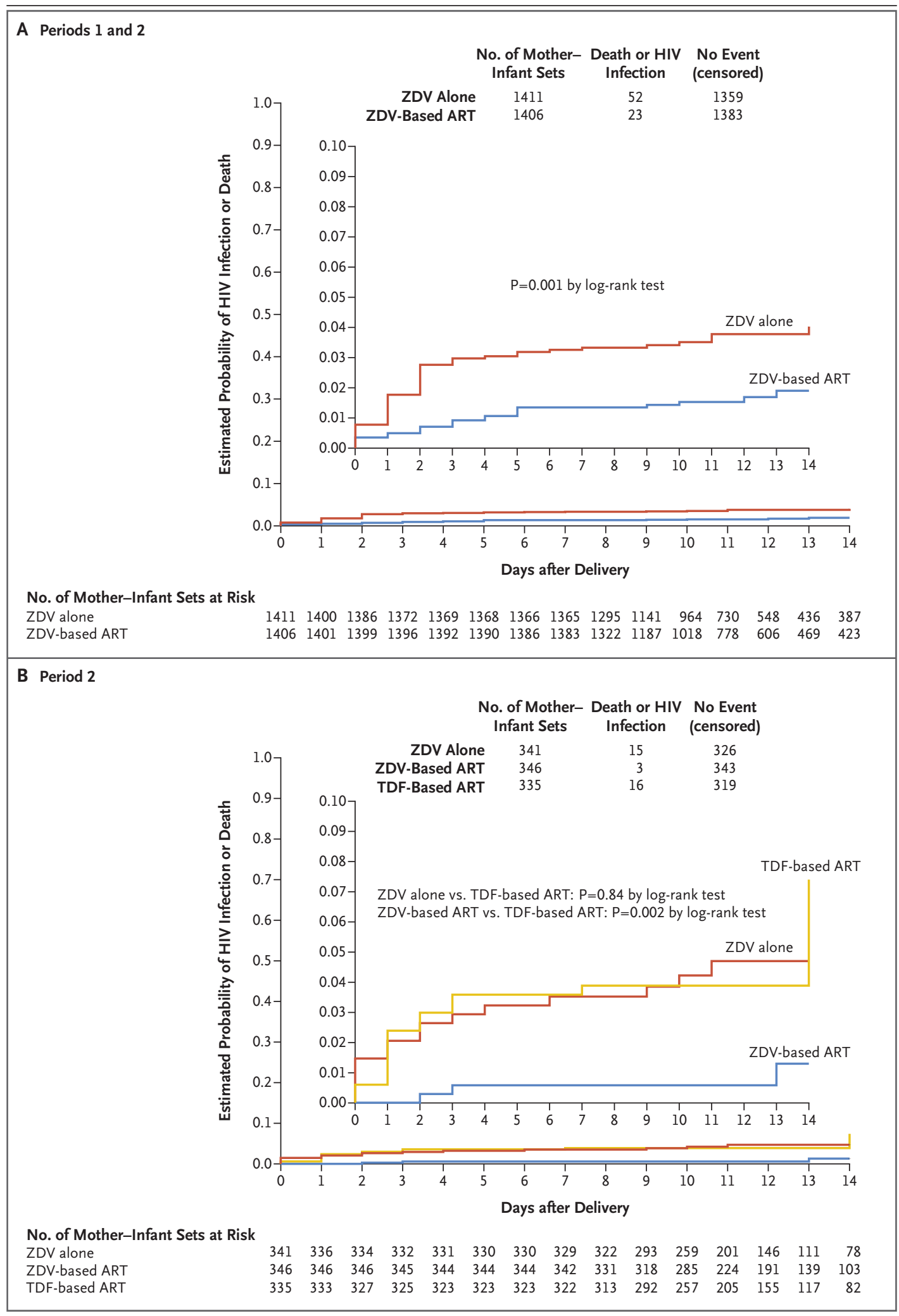

N ENGLJ MED 375;18 NEJM.ORg NOVEMBER 3, 2016

The New England Journal of Medicine 


\begin{tabular}{|c|c|c|c|c|c|c|}
\hline \multirow[t]{3}{*}{ Outcome } & \multicolumn{3}{|c|}{ Antepartum Randomization Group } & \multicolumn{3}{|c|}{ P Value } \\
\hline & $\begin{array}{l}\text { ZDV } \\
\text { Alone }\end{array}$ & $\begin{array}{l}\text { ZDV-Based } \\
\text { ART }\end{array}$ & $\begin{array}{l}\text { TDF-Based } \\
\text { ART }\end{array}$ & $\begin{array}{l}\text { ZDV Alone vs. } \\
\text { ZDV-Based } \\
\text { ART }\end{array}$ & $\begin{array}{l}\text { ZDV Alone vs. } \\
\text { TDF-Based } \\
\text { ART }\end{array}$ & $\begin{array}{c}\text { ZDV-Based } \\
\text { ART vs. TDF- } \\
\text { Based ART }\end{array}$ \\
\hline & \multicolumn{3}{|c|}{ number/total number (percent) } & & & \\
\hline \multicolumn{7}{|l|}{ Maternal adverse events } \\
\hline \multicolumn{7}{|l|}{$\begin{array}{c}\text { Periods } 1 \text { and 2: ZDV alone vs. } \\
\text { ZDV-based ART }\end{array}$} \\
\hline Any grade $\geq 2$ adverse event $†$ & $261 / 1510(17.3)$ & $318 / 1505(21.1)$ & - & 0.008 & & \\
\hline $\begin{array}{l}\text { Grade } \geq 2 \text { abnormal blood } \\
\text { chemical value }\end{array}$ & $19 / 1510(1.3)$ & $88 / 1505(5.8)$ & - & $<0.001$ & & \\
\hline \multicolumn{7}{|l|}{ Period 2 only: all three groups } \\
\hline Any grade $\geq 2$ adverse event $\uparrow$ & $59 / 393(15.0)$ & $61 / 385(15.8)$ & $60 / 380(15.8)$ & & 0.77 & $>0.99$ \\
\hline $\begin{array}{l}\text { Grade } \geq 2 \text { abnormal blood } \\
\text { chemical value }\end{array}$ & $3 / 392(0.8)$ & $18 / 385(4.7)$ & $11 / 380(2.9)$ & & 0.03 & 0.26 \\
\hline \multicolumn{7}{|l|}{ Adverse pregnancy outcomes } \\
\hline \multicolumn{7}{|l|}{$\begin{array}{c}\text { Periods } 1 \text { and 2: ZDV alone vs. } \\
\text { ZDV-based ART }\end{array}$} \\
\hline Any adverse outcomet & $389 / 1414(27.5)$ & $563 / 1407(40.0)$ & - & $<0.001$ & & \\
\hline Low birth weight: $<2500 \mathrm{~g}$ & $161 / 1347(12.0)$ & $306 / 1332(23.0)$ & - & $<0.001$ & & \\
\hline Preterm delivery: $<37 \mathrm{wk}$ & $185 / 1411(13.1)$ & $288 / 1406(20.5)$ & - & $<0.001$ & & \\
\hline \multicolumn{7}{|l|}{ Period 2: all three groups } \\
\hline Any adverse outcome & $91 / 334(27.2)$ & $123 / 328(37.5)$ & $111 / 320(34.7)$ & & 0.04 & 0.46 \\
\hline Low birth weight: $<2500 \mathrm{~g}$ & $28 / 315(8.9)$ & $65 / 319(20.4)$ & $51 / 301(16.9)$ & & 0.004 & 0.30 \\
\hline Preterm delivery: $<37 \mathrm{wk}$ & $46 / 341(13.5)$ & $68 / 346(19.7)$ & $62 / 335(18.5)$ & & 0.09 & 0.77 \\
\hline \multicolumn{7}{|l|}{ Severe adverse pregnancy outcomes } \\
\hline \multicolumn{7}{|l|}{$\begin{array}{c}\text { Periods } 1 \text { and 2: ZDV alone vs. } \\
\text { ZDV-based ART }\end{array}$} \\
\hline Any severe adverse outcome $\mathbb{}$ & $83 / 1399(5.9)$ & $99 / 1385(7.1)$ & - & 0.22 & & \\
\hline Very preterm delivery: $<34 \mathrm{wk}$ & $37 / 1411(2.6)$ & $44 / 1406(3.1)$ & - & 0.43 & & \\
\hline Infant deaths through wk 1 & $28 / 1432(2.0)$ & $17 / 1419(1.2)$ & - & 0.13 & & \\
\hline \multicolumn{7}{|l|}{ Period 2 only: all three groups } \\
\hline Any severe adverse outcome $\mathbb{}$ & $22 / 329(6.7)$ & $14 / 322(4.3)$ & $29 / 314(9.2)$ & & 0.25 & 0.02 \\
\hline Very preterm delivery: $<34 \mathrm{wk}$ & $11 / 341(3.2)$ & $9 / 346(2.6)$ & $20 / 335(6.0)$ & & 0.10 & 0.04 \\
\hline Infant deaths through wk 1 & $11 / 349(3.2)$ & $2 / 346(0.6)$ & $15 / 341(4.4)$ & & 0.43 & 0.001 \\
\hline
\end{tabular}

* For more details on maternal and infant safety, see Tables S2 through S7 in the Supplementary Appendix.

$\uparrow$ The composite outcome was grade 2 or higher hematologic abnormalities or abnormal blood chemical values or grade 3 or higher signs or symptoms during pregnancy through week 1 post partum.

$\Varangle$ The composite outcome was a low birth weight of less than $2500 \mathrm{~g}$, a preterm delivery before 37 weeks of gestation (according to the Ballard examination, when available, or obstetrical estimate), spontaneous abortion ( $<20$ weeks of gestation), stillbirth ( $\geq 20$ weeks of gestation), or a congenital anomaly (according to the criteria of the Metropolitan Atlanta Congenital Defects Program of the Centers for Disease Control and Prevention).

$\int$ Severe adverse pregnancy outcomes included a very low birth weight of less than $1500 \mathrm{~g}$, a very preterm delivery before 34 weeks, spontaneous abortion, stillbirth, or a major congenital anomaly.

based ART, $80 \%$ of very preterm deliveries occurred during period 1 , with only $20 \%$ during period 2 .

\section{Infant Safety Outcomes}

Among 3202 live births, no significant differences were observed in pairwise comparisons of the groups for composite or individual infant 
adverse outcomes other than death (Table 3, and Tables S5 and S6 in the Supplementary Appendix). There were 60 infant deaths (1.9\% of live births) by week 1 (Table 3; Table S7 in the Supplementary Appendix lists reported causes). No significant difference in infant deaths was observed between the group assigned to zidovudine-based ART and the zidovudine-alone group (periods 1 and 2$)(1.2 \%$ and $2.0 \%$, respectively; $\mathrm{P}=0.13)$. During period 2, no significant difference in infant deaths was observed between the group assigned to tenofovir-based ART and the zidovudine-alone group $(4.4 \%$ and $3.2 \%$, respectively; $\mathrm{P}=0.43$ ), but the group assigned to tenofovirbased ART had significantly more infant deaths than the group assigned to zidovudine-based ART $(4.4 \%$ vs. $0.6 \%, \mathrm{P}<0.001)$. However, of 17 deaths in the group assigned to zidovudine-based ART, 88\% (15 deaths) occurred during period 1 and only $12 \%$ ( 2 deaths) during period 2 . A post hoc analysis suggested that mortality by week 1 was highest among very preterm infants (27.0\%), with lower mortality rates among those born at a gestational age of 34 to less than 37 weeks (3.3\%) and those born at term (0.5\%).

\section{DISCUSSION}

The PROMISE trial showed the efficacy of tripledrug ART for preventing transmission among HIV-infected pregnant women with high CD4 counts. All three regimens studied had a transmission rate of less than $2 \%$, but the ART regimens had significantly lower rates of transmission than zidovudine alone $(0.5 \%$ vs. $1.8 \%)$. However, both ART regimens were associated with higher rates of adverse events than zidovudine alone.

Maternal grade 2 or higher laboratory abnormalities were more common with zidovudinebased ART (periods 1 and 2) and tenofovir-based ART (period 2) than with zidovudine alone. However, no significant difference between the two ART regimens was observed. Specifically, there were no increases in hematologic toxic effects with zidovudine-based ART or renal toxic effects with tenofovir-based ART, and there were no maternal deaths. Rates of trial-drug discontinuation due to toxic effects were low.

Higher rates of adverse pregnancy outcomes were observed with zidovudine-based ART (periods 1 and 2) and tenofovir-based ART (period 2) than with zidovudine alone, but no significant difference between the two ART regimens was observed. Our results are consistent with those of observational studies in Africa that controlled for CD4 count and showed higher rates of preterm delivery and low birth weight among women initiating ART (primarily nevirapine-based) during pregnancy than among those starting zidovudine plus single-dose nevirapine. ${ }^{5,6}$ In resourcelimited settings, preterm delivery and low birth weight can be associated with increased morbidity and increased newborn or later postnatal mortality. ${ }^{8-11}$

Reassuringly, the rate of very preterm delivery did not differ significantly between the zidovudine-alone group and the group assigned to zidovudine-based ART (periods 1 and 2) or the group assigned to tenofovir-based ART (period 2). However, the rate of very preterm delivery differed significantly between the two ART groups during period 2 (2.6\% with zidovudine-based ART vs. $6.0 \%$ with tenofovir-based ART, $\mathrm{P}=0.04$ ), as did early infant mortality (correlated with very preterm delivery) $(0.6 \%$ vs. $4.4 \%, \mathrm{P}<0.001)$. This resulted in a significantly higher rate of HIV-free survival among infants in the group assigned to zidovudine-based ART than among those in the group assigned to tenofovir-based ART during period 2 (but no significant difference between infants in the group assigned to tenofovir-based ART and those in the zidovudine-alone group).

The reason for differences in very preterm delivery and infant mortality between the two ART groups is unclear. Comparisons are limited to concurrent randomization during period 2 . Overall, 40 of 101 very preterm deliveries (40\%) and 28 of 60 infant deaths (47\%) occurred during period 2. However, in the group assigned to zidovudine-based ART, $80 \%$ of very preterm deliveries and $88 \%$ of infant deaths occurred during period 1 . This suggests there could be some unknown confounder resulting in lower rates of very preterm delivery and infant death in the group assigned to zidovudine-based ART during period 2. A study in Botswana that compared birth outcomes in women receiving tenofovirbased ART (with efavirenz) or zidovudine-based ART (primarily with nevirapine) showed no significant differences in preterm delivery or stillbirth. ${ }^{12}$ A potential biologic cause may be a pharmacokinetic interaction between lopinavirritonavir and tenofovir; decreased renal clearance 
of tenofovir and increased plasma and intracellular levels, particularly in women, have been reported with concomitant administration. ${ }^{13-15}$ In the PROMISE trial, the lopinavir-ritonavir dose was increased during the third trimester because pharmacokinetic studies showed decreased lopinavir-ritonavir levels with standard doses in late pregnancy. ${ }^{16,17}$

The ART regimens in the PROMISE trial were protease inhibitor-based because nevirapine-based ART was contraindicated in women with a CD4 count of more than 250 cells per cubic millimeter, and efavirenz, the other available nonnucleoside reverse-transcriptase inhibitor (NNRTI), was contraindicated in pregnancy at that time. There are inconsistent findings regarding protease inhibitor-based ART and preterm delivery. ${ }^{3,18-21}$ Some studies have shown that protease inhibitors reduce progesterone production in the trophoblast in vitro and that low progesterone levels are associated with preterm delivery. ${ }^{22-25}$ However, other studies have shown that nevirapine-based ART is also associated with a higher rate of preterm delivery than zidovudine plus single-dose nevirapine. ${ }^{5,6}$ The PROMISE trial was not designed to compare protease inhibitor-based ART with NNRTI-based ART, and we cannot comment on whether the current WHO-recommended ART regimen of tenofovir, emtricitabine, and efavirenz would be associated with a lower risk of low birth weight or preterm delivery than the ART regimens in the PROMISE trial. However, the Botswana data are reassuring in that the combination of tenofovir, emtricitabine, and efavirenz was not associated with higher rates of adverse pregnancy outcomes than zidovudine-based ART. ${ }^{12}$

In conclusion, the PROMISE trial showed superior efficacy of triple-drug ART, as compared with zidovudine plus single-dose nevirapine, for the prevention of mother-to-child transmission in HIV-infected women with high CD4 cell counts but also showed higher rates of adverse events. On the basis of recent trials, the WHO recommends ART for HIV-infected persons regardless of $\mathrm{CD} 4$ cell count, and there are clear benefits of ART for the prevention of mother-to-child transmission and maternal health. ${ }^{26,27}$ However, it is also clear that the most efficacious and safest triple-drug ART regimens during pregnancy remain to be defined. Our findings emphasize the need for continued research to assess ART in pregnancy to ensure safer pregnancies for HIVinfected women and healthier outcomes for their uninfected infants.

The views expressed in this article are solely those of the authors and do not necessarily represent the official views of the National Institutes of Health (NIH).

Supported by the National Institute of Allergy and Infectious Diseases of the NIH under award numbers UM1AI068632 (IMPAACT LOC), UM1AI068616 (IMPAACT SDMC), and UM1AI106716 (IMPAACT LC), with cofunding from the Eunice Kennedy Shriver National Institute of Child Health and Human Development and the National Institute of Mental Health.

Dr. Violari reports receiving consulting fees from Janssen and grant support from Gilead Sciences and ViiV Healthcare, all paid to her institution. No other potential conflict of interest relevant to this article was reported.

Disclosure forms provided by the authors are available with the full text of this article at NEJM.org.

We thank the mothers and their infants who participated in the trial, as well as the PROMISE staff at the clinical research sites; the staff at the IMPAACT central laboratory at the University of North Carolina at Chapel Hill; the statistical staff at the Center for Biostatistics in AIDS Research, Harvard T.H. Chan School of Public Health; and the data-management staff at the Frontier Science and Technology Research Foundation.

We dedicate this article to Dr. Edward Handelsman (Division of AIDS, NIH), Dr. Stephen Lagakos (Center for Biostatistics in AIDS Research, Harvard T.H. Chan School of Public Health), and Mrs. Linda Millar (Frontier Science and Technology Research Foundation), in grateful memory of their many contributions to the PROMISE 1077 trial and HIV-AIDS research.

\section{APPENDIX}

The authors' full names and academic degrees are as follows: Mary G. Fowler, M.D., M.P.H., Min Qin, Ph.D., Susan A. Fiscus, Ph.D., Judith S. Currier, M.D., Patricia M. Flynn, M.D., Tsungai Chipato, M.B., Ch.B., M.C.E., James McIntyre, F.R.C.O.G., Devasena Gnanashanmugam, M.D., George K. Siberry, M.D., M.P.H., Anne S. Coletti, M.S., Taha E. Taha, M.D., Ph.D., Karin L. Klingman, M.D., Francis E. Martinson, M.B., Ch.B., Ph.D., Maxensia Owor, M.B., Ch.B., M.P.H., Avy Violari, M.D., Dhayendre Moodley, Ph.D., Gerhard B. Theron, M.D., Ramesh Bhosale, M.D., Raziya Bobat, M.B., Ch.B., M.D., Benjamin H. Chi, M.D., Renate Strehlau, M.B., Ch.B., Pendo Mlay, M.D., Amy J. Loftis, B.S., Renee Browning, R.N., M.S.N., Terence Fenton, Ed.D., Lynette Purdue, Pharm.D., Michael Basar, B.A., David E. Shapiro, Ph.D., and Lynne M. Mofenson, M.D.

The authors' affiliations are as follows: the Department of Pathology, Johns Hopkins University School of Medicine (M.G.F.), and the Department of Epidemiology, Johns Hopkins University Bloomberg School of Public Health (T.E.T.), Baltimore, and the Division of AIDS, National Institute of Allergy and Infectious Diseases (D.G., K.L.K., R. Browning, L.P.), and the Eunice Kennedy Shriver National Institute of Child Health and Human Development (G.K.S.), National Institutes of Health, Bethesda - all in Maryland; the Center for Biostatistics in AIDS Research, Harvard T.H. Chan School of Public Health, Boston (M.Q., T.F., D.E.S.); the Retrovirology Core Laboratory, University of North Carolina School of Medicine, Chapel Hill (S.A.F., A.J.L.), and FHI 360, Durham (A.S.C.) - both in North Carolina; the UCLA Center for Clinical AIDS Research and Education, Los Angeles (J.S.C.); St. Jude Children's Research Hospital, Memphis, TN (P.M.F.); the Department of Obstetrics and Gynecology, College of Health Sciences, University of Zimbabwe, Harare 
(T.C.); Anova Health Institute (J.M.), Perinatal HIV Research Unit, University of the Witwatersrand, Faculty of Health Sciences (A.V.), and Empilweni Services and Research Unit (R.S.), Johannesburg, School of Public Health and Family Medicine, University of Cape Town (J.M.), and the Department of Obstetrics and Gynaecology, Stellenbosch University (G.B.T.), Cape Town, and the Centre for the AIDS Programme of Research in South Africa-Umlazi Clinical Research Site (D.M.) and Durban Paediatric HIV (R. Bobat), Nelson R. Mandela School of Medicine, University of KwaZulu-Natal, Durban — all in South Africa; the University of North Carolina Project, Kamuzu Central Hospital-Tidziwe Center, Lilongwe, Malawi (F.E.M.); Makerere University-Johns Hopkins University Research Collaboration, Kampala, Uganda (M.O.); the Department of Obstetrics and Gynecology, B.J. Medical College, Pune, India (R. Bhosale); the Centre for Infectious Disease Research in Zambia, Lusaka, Zambia (B.H.C.); Kilimanjaro Christian Medical Center-Duke University Collaboration, Moshi, Tanzania (P.M.); Frontier Science and Technology Research Foundation, Amherst, NY (M.B.); and the Elizabeth Glaser Pediatric AIDS Foundation, Washington, DC (L.M.M.).

\section{REFERENCES}

1. Connor EM, Sperling RS, Gelber R, et al. Reduction of maternal-infant transmission of human immunodeficiency virus type 1 with zidovudine treatment. N Engl J Med 1994;331:1173-80.

2. Panel on Treatment of HIV-Infected Pregnant Women and Prevention of Perinatal Transmission. Recommendations for use of antiretroviral drugs in pregnant HIV-1-infected women for maternal health and interventions to reduce perinatal HIV transmission in the United States. Rockville, MD: AIDSinfo, 2016 (http://aidsinfo .nih.gov/contentfiles/lvguidelines/Perinatal GL.pdf).

3. Mofenson LM. Antiretroviral therapy and adverse pregnancy outcome: the elephant in the room? J Infect Dis 2016;213: 1051-4.

4. Chen JY, Ribaudo HJ, Souda S, et al. Highly active antiretroviral therapy and adverse birth outcomes among HIV-infected women in Botswana. J Infect Dis 2012; 206:1695-705.

5. Li N, Sando MM, Spiegelman D, et al. Antiretroviral therapy in relation to birth outcomes among HIV-infected women: a cohort study. J Infect Dis 2016;213:105764.

6. Antiretroviral drugs for treating pregnant women and preventing HIV infection in infants: recommendations for a public health approach. Geneva: World Health Organization, 2010 (http://whqlibdoc.who .int/publications/2010/9789241599818_eng .pdf?ua=1).

7. Department of Health and Human Services, National Institutes of Health, National Institute of Allergy and Infectious Diseases, Division of AIDS. Division of AIDS table for grading the severity of adult and pediatric adverse events, version 1.0. August 2009 (http://rsc.tech-res.com/ docs/default-source/safety/table_for_ grading_severity_of_adult_pediatric_ adverse_events.pdf).

8. Marchant T, Willey B, Katz J, et al. Neonatal mortality risk associated with preterm birth in East Africa, adjusted by weight for gestational age: individual participant level meta-analysis. PLoS Med 2012;9(8):e1001292.
9. Liu L, Johnson HL, Cousens S, et al. Global, regional, and national causes of child mortality: an updated systematic analysis for 2010 with time trends since 2000. Lancet 2012;379:2151-61.

10. Katz J, Lee AC, Kozuki N, et al. Mortality risk in preterm and small-for-gestational-age infants in low-income and middle-income countries: a pooled country analysis. Lancet 2013;382:417-25.

11. Taha TE, Dadabhai SS, Sun J, Rahman MH, Kumwenda J, Kumwenda N. Child mortality levels and trends by HIV status in Blantyre, Malawi: 1989-2009. J Acquir Immune Defic Syndr 2012;61:226-34.

12. Zash R, Souda S, Chen JY, et al. Reassuring birth outcomes with tenofovir/ emtricitabine/efavirenz used for prevention of mother-to-child transmission of HIV in Botswana. J Acquir Immune Defic Syndr 2016;71:428-36.

13. Kiser JJ, Carten ML, Aquilante CL, et al. The effect of lopinavir/ritonavir on the renal clearance of tenofovir in HIV-infected patients. Clin Pharmacol Ther 2008;83: 265-72.

14. Kearney BP, Mathias A, Mittan A, Sayre J, Ebrahimi R, Cheng AK. Pharmacokinetics and safety of tenofovir disoproxil fumarate on coadministration with lopinavir/ritonavir. J Acquir Immune Defic Syndr 2006;43:278-83.

15. Pruvost A, Negredo E, Théodoro F, et al. Pilot pharmacokinetic study of human immunodeficiency virus-infected patients receiving tenofovir disoproxil fumarate (TDF): investigation of systemic and intracellular interactions between TDF and abacavir, lamivudine, or lopinavir-ritonavir. Antimicrob Agents Chemother 2009; 53:1937-43.

16. Stek AM, Mirochnick M, Capparelli E, et al. Reduced lopinavir exposure during pregnancy. AIDS 2006;20:1931-9.

17. Mirochnick M, Best BM, Stek AM, et al. Lopinavir exposure with an increased dose during pregnancy. J Acquir Immune Defic Syndr 2008;49:485-91.

18. Watts DH, Williams PL, Kacanek D, et al. Combination antiretroviral use and preterm birth. J Infect Dis 2013;207:61221.
19. Kourtis AP, Fowler MG. Antiretroviral use during pregnancy and risk of preterm delivery: more questions than answers. J Infect Dis 2011;204:493-4.

20. Sibiude J, Warszawski J, Tubiana R, et al. Premature delivery in HIV-infected women starting protease inhibitor therapy during pregnancy: role of the ritonavir boost? Clin Infect Dis 2012;54:134860.

21. Powis KM, Kitch D, Ogwu A, et al. Increased risk of preterm delivery among HIV-infected women randomized to protease versus nucleoside reverse transcriptase inhibitor-based HAART during pregnancy. J Infect Dis 2011;204:506-14.

22. Papp E, Mohammadi H, Loutfy MR, et al. HIV protease inhibitor use during pregnancy is associated with decreased progesterone levels, suggesting a potential mechanism contributing to fetal growth restriction. J Infect Dis 2015;211: 10-8.

23. Papp E, Balogun $\mathrm{K}$, Banko $\mathrm{N}$, et al. Low prolactin and high 20- $\alpha$-hydroxysteroid dehydrogenase levels contribute to lower progesterone levels in HIV-infected pregnant women exposed to protease inhibitor-based combination antiretroviral therapy. J Infect Dis 2016;213:153240.

24. Powis KM, Shapiro RL. Protease inhibitors and adverse birth outcomes: is progesterone the missing piece to the puzzle? J Infect Dis 2015;211:4-7.

25. Caritis SN, Venkataramanan R, Thom $\mathrm{E}$, et al. Relationship between 17-alpha hydroxyprogesterone caproate concentration and spontaneous preterm birth. Am J Obstet Gynecol 2014;210(2):128.e1-6.

26. The INSIGHT START Study Group. Initiation of antiretroviral therapy in early asymptomatic HIV infection. N Engl J Med 2015;373:795-807.

27. Guideline on when to start antiretroviral therapy and on pre-exposure prophylaxis for HIV. Geneva: World Health Organization, September 2015 (http://www .who.int/hiv/pub/guidelines/earlyrelease-arv/ en/).

Copyright (C) 2016 Massachusetts Medical Society. 